\title{
What Words Should We Use When Discussing Excess Weight?
}

\author{
Gareth R. Dutton, PhD, Fei Tan, PhD, Michael G. Perri, PhD, Curtis C. Stine, MD, \\ Melissa Dancer-Brown, RD, Mary Goble, MSN, and Nancy Van Vessem, MD
}

Background: There is limited research on how patients prefer physicians to communicate about the topic of obesity, and there is even less understanding of which terms physicians most commonly use.

Methods: In this cross-sectional, nonrandom sampling study, patients who were seeking treatment for weight loss rated the desirability of 12 terms to describe excess weight, and physicians rated the likelihood with which they would use those terms during clinical encounters. Participants rated terms on a 5-point scale, with -2 representing "very undesirable" or "definitely would not use" and +2 representing "very desirable" or "definitely would use."

Results: Patients $\left(\mathrm{n}=143\right.$; mean age, 46.8 years; mean body mass index, $\left.36.9 \mathrm{~kg} / \mathrm{m}^{2}\right)$ rated "weight" (mean \pm SD) as the most desirable term $(1.13 \pm 1.10)$, although it did not significantly differ from 5 other terms provided. They rated "fatness" $(-1.30 \pm 1.22)$ as the most undesirable term, although this rating did not differ significantly from 4 other terms. Physicians affiliated with a community-based medical school $(n=108$; mean age, 48.8 years; $79.6 \%$ primary care specialty) were most likely to use "weight" $(1.42 \pm 0.89)$, which was significantly different from ratings for all other terms. They were least likely to use "fatness" $(-1.74 \pm 0.59)$, although this rating did not differ significantly from 3 other terms.

Conclusion: Physicians generally reported that they use terminology that patients had rated more favorably, and they tend to avoid terms that patients may find undesirable. Understanding the preferences and terminology used by patients and physicians is an important initial step to ensure that communications related to obesity and weight loss are efficient and effective. ( $\mathrm{J}$ Am Board Fam Med 2010; 23:606-613.)

Keywords: Obesity, Physician-Patient Relations, Primary Health Care, Communication, Medical Terminology

The provision of weight loss counseling by physicians in primary care settings offers a potentially effective approach to combating the problem of overweight and obesity. ${ }^{1-4}$ However, physicians often neglect broaching the topics of obesity and weight loss during primary care encounters. ${ }^{3,5-8}$ Barriers that may hinder physicians' provision of

This article was externally peer reviewed.

Submitted 4 February 2010; revised 19 May 2010; accepted 24 May 2010.

From the Florida State University College of Medicine (GRD, CCS) and the Florida Agricultural and Mechanical University (FT), Tallahassee; the Capital Health Plan, Tallahassee, FL (MD-B, MG, NVV); and the University of Florida, Gainesville (MGP).

Funding: Financial support for this project was provided by the Florida State University College of Medicine. In-kind services were provided by Capital Health Plan.

Conflict of interest: none declared.

Corresponding author: Gareth R. Dutton, PhD, Florida State University College of Medicine, Department of Medical Humanities and Social Sciences, 1115 W. Call St., Tallahassee, FL 32306 (E-mail: gareth.dutton@med.fsu.edu). weight-loss counseling include time constraints, lack of reimbursement, and perceptions that treatment will be ineffective. In addition, physicians may feel ill-equipped to address these issues. ${ }^{5,9-12}$

Despite these barriers, overweight and obese patients may desire greater involvement of their primary care physician in weight-loss counseling and treatment efforts. ${ }^{6,7}$ Until recently, however, few studies have examined how patients would prefer their physicians to discuss the topic of obesity. In one study, obese patients rated the term "weight" as significantly more desirable than a variety of terms physicians could use to describe excess weight; they rated "obesity," "excess fat," and "fatness" as the most undesirable terms physicians could use. ${ }^{13}$ The parents of pediatric patients overwhelmingly preferred that physicians use the phrase "gaining too much weight" as compared with other options, including "overweight," when discussing their child's weight. ${ }^{14}$ Use of shared ter- 
minology that is agreeable and understandable for both patients and physicians is important for ensuring that effective communication occurs during the clinical encounter. ${ }^{15}$

Although previous work suggests that patients prefer some terms used by physicians over others, there is less information available regarding what terms physicians use during clinical encounters. Among a small sample $(n=19)$ of general practitioners, Tailor and Ogden ${ }^{16}$ found that physicians were more likely to use a euphemism with patients (eg, "Your weight may be damaging your health.") than the term "obesity." Understanding what terms physicians use may be particularly helpful because these word choices may influence patients' responses. For instance, patients with a body mass index (BMI) $<30 \mathrm{~kg} / \mathrm{m}^{2}$ found physicians' use of the term "obesity" more emotionally distressing, whereas obese patients (ie, BMI $>30 \mathrm{~kg} / \mathrm{m}^{2}$ ) found the euphemism "your weight may be damaging your health" more distressing. ${ }^{16}$

In addition, research indicates that physicians are not immune to the negative, implicit biases held against overweight and obese individuals. ${ }^{10,17}$ Also, physicians may spend less time with obese patients than with normal weight patients. ${ }^{18}$ Given these findings, it is worthwhile to explore whether physicians explicitly endorse the use of potentially derogatory terms to describe excess weight. Furthermore, previous research on patient preferences only included obese and severely obese individuals; there is a lack of information about the preferences of overweight patients (ie, those with a BMI between 25 and $29.9 \mathrm{~kg} / \mathrm{m}^{2}$ ). There is also limited research about physician preferences for communication about excess weight. Therefore, the purpose of this study was to compare which terms overweight and obese patients prefer to describe excess weight. We also investigated the reported practices of primary care physicians when addressing this topic with patients.

\section{Methods \\ Participants}

The patients who were recruited to participate in this study were members of a managed care organization (MCO) who were seeking treatment for weight loss. The MCO provides clinical services for approximately 110,000 members who receive health benefits and coverage through numerous employer groups and Medicare. The MCO offers group-based behavioral weight loss treatment for interested members, and these members were targeted for study recruitment. Inclusion criteria consisted of (1) attendance to an initial informational session regarding the weight-loss program, and (2) being overweight or obese (ie, BMI $>25 \mathrm{~kg} / \mathrm{m}^{2}$ ). Participants' data were excluded if their BMI was $<25 \mathrm{~kg} / \mathrm{m}^{2}$ or if they did not provide adequate information needed to calculate BMI.

The physicians who were recruited to participate in this study were family medicine and internal medicine faculty of a community-based medical school. Because of the medical school's organizational structure, the sample was made up of physicians who were primarily engaged in direct provision of outpatient clinical services in the community. Although some clinical faculty members were providers within the managed care network, physician recruitment was not limited to this organization. Inclusion criteria consisted of having a clinical faculty appointment with the medical school and having an affiliation with the family medicine or internal medicine clerkships.

\section{Procedure}

The study protocol was approved by the institutional review boards of the participating organizations.

\section{Patients}

The MCO provides monthly informational sessions about the group-based weight-loss intervention to members who are interested in the program, and members attending these monthly sessions were targeted for study recruitment. Recruitment occurred during an 8-month period. Patients completed the survey in the clinic before the initial weight-loss session.

\section{Physicians}

Physicians received an email invitation that included an electronic link to the survey. A reminder email that also included the survey link was sent to physicians approximately 2 to 3 weeks after the initial request. Physicians completed the survey online.

\section{Measures}

Both patients and physicians completed anonymous, self-report surveys that had been modified 
from validated questionnaires used in previous studies. ${ }^{13}$ Surveys included demographic information, the Weight Preferences Questionnaire, and measurements of height and weight.

\section{Demographic and Background Information}

Background information collected from physicians included age, sex, clinical specialty, primary clinical setting (inpatient or outpatient), and years in practice. Demographic characteristics requested from patients included age, sex, ethnicity, marital status, and level of education.

\section{Weight Preferences Questionnaire}

All participants completed the Weight Preferences Questionnaire, which was developed and validated in previous research with obese patients. ${ }^{13}$ This survey provided respondents with a hypothetical clinical scenario of a patient who was visiting his or her physician, with the topic of weight introduced during the visit. Using a 5-point Likert scale, patients were asked to rate the desirability of 12 terms the physician could use to describe weight $(1=$ very desirable; $5=$ very undesirable).

The response format for physicians was modified slightly. Using a 5-point Likert scale, physicians were asked to rate the likelihood that he or she would use each of the 12 descriptors with a patient $(1=$ definitely not use; $5=$ definitely use). The terms included (1) weight, (2) heaviness, (3) obesity, (4) BMI, (5) excess weight, (6) fatness, (7) excess fat, (8) large size, (9) unhealthy body weight, (10) weight problem, (11) unhealthy BMI, and (12) overweight status. The first 11 terms were identical to the ones used in the initial version of this measure, ${ }^{13}$ although "overweight status" was added to have at least one term that incorporated "overweight" into the descriptor. To facilitate ease of comparisons between the ratings of patients and physicians, values were transformed to consistent scales ranging from -2 ("very undesirable" or "definitely not use") to +2 ("very desirable" or "definitely use").

\section{Height and Weight}

Patients and physicians were asked to provide their height (in feet and inches) and current weight (in pounds). Self-reports of these anthropometric measures have been included in previous surveys related to weight loss ${ }^{19}$ and have been shown to be a valid and reliable approach that corresponds with objective, documented measurements. ${ }^{20,21}$

\section{Statistical Analyses}

For physicians and patients, separate multivariate analysis of variance was used to assess significant differences in the mean scores of the 12 weight terms. Multivariate analyses of variance for the physician and patient datasets were both significant $(P<.0001)$. After these significant omnibus tests, Tukey's multiple comparison procedure was applied separately to both datasets to rank and compare the 12 terms for each group. In other words, simultaneous 95\% CIs based on Tukey's method were calculated for physicians and patients to generate an overall significance level of .05 for each family of comparisons and thereby account for multiple comparisons within each group.

In addition, physicians were categorized by their weight status (ie, BMI $<25.0 \mathrm{~kg} / \mathrm{m}^{2}$ or $\mathrm{BMI} \geq 25.0$ $\mathrm{kg} / \mathrm{m}^{2}$ ) to enable comparison of each word's mean ratings between normal-weight and overweight/ obese physicians. For this purpose, 12 analysis of variance models were used; each model's significance level was set to be .004 to control for multiple comparisons. Similarly, analyses of patient subgroups were conducted by comparing overweight and obese patients' ratings. African-American and white patients mean scores for each term were also compared. The significance level of each individual analysis of variance model in these patient comparisons was .004 to account for multiple comparisons.

\section{Results}

\section{Sample Characteristics}

Recruitment and retention for both samples (physicians and patients) are summarized in Figure 1. The survey response rate among patients was $98 \%$. The response rate among physicians was $24.4 \%$. The assessed demographic characteristics for patients and physicians are summarized in Table 1.

\section{Weight Terminology Preferences Patients}

There was considerable overlap in patients' ratings of the desirability of different words. "Weight" (mean $\pm \mathrm{SD}$ ) was rated by patients as the most desirable choice for physicians to use $(1.13 \pm 1.10)$ and was the only word rated in the range of "desirable" to "very desirable." However, it did not 
Figure 1. Participant recruitment and retention. BMI, body mass index.

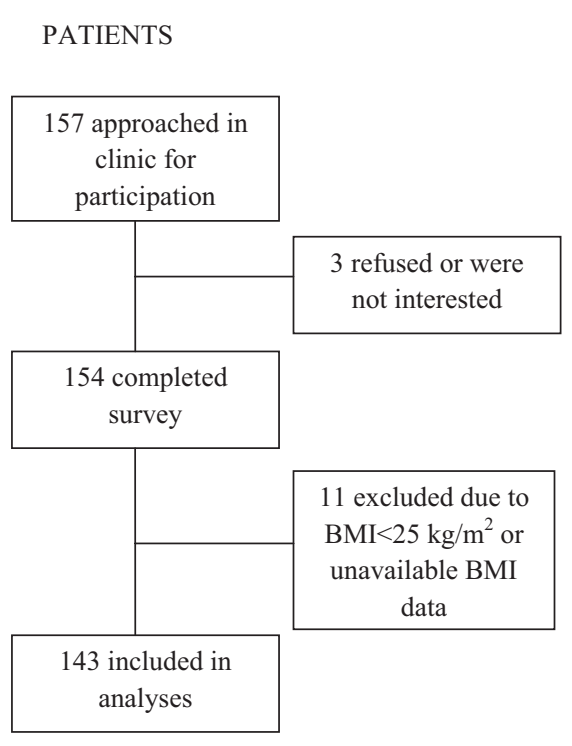

differ significantly from 5 other words that were rated in the range of "desirable" to "neutral," including "BMI" $(0.70 \pm 1.26)$, "unhealthy body weight" (0.46 \pm 1.35$)$, "unhealthy BMI" $(0.42 \pm$ $1.30)$, "weight problem" $(0.28 \pm 1.33)$, and "excess weight" $(0.20 \pm 1.31)$. The term "fatness" $(-1.30 \pm 1.22)$ was rated by patients as least desirable, although this rating was not significantly different from patients' ratings of "excess fat"

Table 1. Characteristics of Patient and Physician Samples

\begin{tabular}{|c|c|c|c|}
\hline & \multicolumn{2}{|c|}{$\begin{array}{c}\text { Patients } \\
(\mathrm{n}=143) \\
(\text { Mean } \pm \mathrm{SD})\end{array}$} & $\begin{array}{c}\text { Physicians } \\
(\mathrm{n}=108) \\
(\text { Mean } \pm \mathrm{SD})\end{array}$ \\
\hline \multicolumn{4}{|l|}{ Weight (kg) } \\
\hline Women & \multicolumn{2}{|c|}{$99.3 \pm 22.4$} & $68.0 \pm 16.5$ \\
\hline Men & \multicolumn{2}{|c|}{$118.3 \pm 27.0$} & $82.6 \pm 14.8$ \\
\hline $\mathrm{BMI}\left(\mathrm{kg} / \mathrm{m}^{2}\right)$ & \multicolumn{2}{|c|}{$36.9 \pm 7.4$} & $25.4 \pm 4.2$ \\
\hline Age (years) & \multicolumn{2}{|c|}{$46.8 \pm 12.5$} & $48.8 \pm 8.5$ \\
\hline \multirow[t]{2}{*}{ Years in practice } & \multicolumn{2}{|c|}{ NA } & $18.3 \pm 9.6$ \\
\hline & & $\mathrm{n}(\%)$ & n (\%) \\
\hline Female & & $128(89.5)$ & $26(24.1)$ \\
\hline Married & & $88(62.4)$ & NA \\
\hline White & & $91(64.5)$ & NA \\
\hline College graduate & & $76(58.9)$ & NA \\
\hline Family or interna & cine & NA & $82(76.0)$ \\
\hline Predominantly ou & ht care & NA & $94(87.0)$ \\
\hline
\end{tabular}

BMI, body mass index.
PHYSICIANS

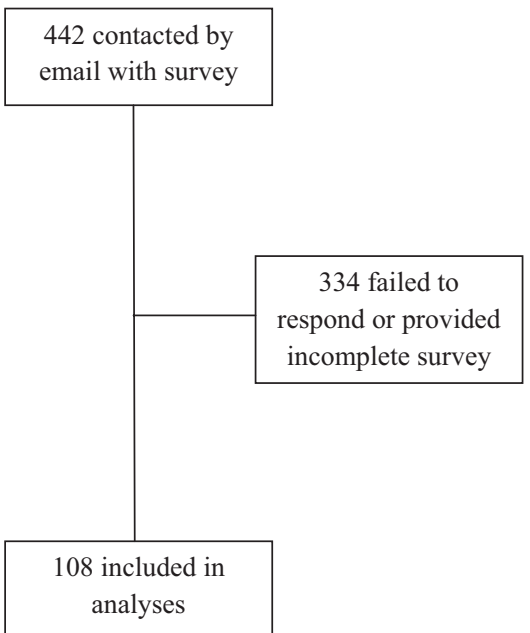

$(-1.06 \pm 1.28)$, "large size" $(-0.99 \pm 1.28)$, "obesity" $(-0.85 \pm 1.35)$, or "heaviness" $(-0.69 \pm$ 1.24). Comparisons of patient ratings for all 12 terms are summarized in Figure 2. In the figure, terms associated with the same letter (a, b, c, etc) do not differ from each other, whereas different letters represent significantly different ratings for those terms.

Approximately $80 \%$ of patients met criteria for obesity (ie, BMI $\geq 30 \mathrm{~kg} / \mathrm{m}^{2}$ ), whereas $20 \%$ were overweight (ie, BMI = 25.0 to $29.9 \mathrm{~kg} / \mathrm{m}^{2}$ ). Overweight and obese patients' preferences for different words were compared, and there was no difference in patients' ratings of desirability for the 12 terms. The ratings of white and African-American patients were compared as well. White patients rated the term "obesity" as significantly more undesirable $(-1.08 \pm 1.22)$ than did African-American patients $(-0.31 \pm 1.52)(P<.003)$. There were no other differences in the preferences of white and AfricanAmerican patients.

\section{Physicians}

Physicians reported that they would "probably" or "definitely" use the term "weight" with patients during clinical encounters. The likelihood of using "weight" $(1.42 \pm 0.89)$ was significantly greater than the reported likelihood of the use of all other terms provided, according to Tukey's simultaneous 95\% CIs. Other terms potentially used by physi- 
Figure 2. Patients' rated desirability of weight-related terms (scores range from -2 [very undesirable] to +2 [very desirable]). Different letters indicate significant differences between terms using Tukey's multiple comparison procedure to maintain an overall family-wise significance level of .05 . Terms with common letters are not significantly different from each other. BMI, body mass index.

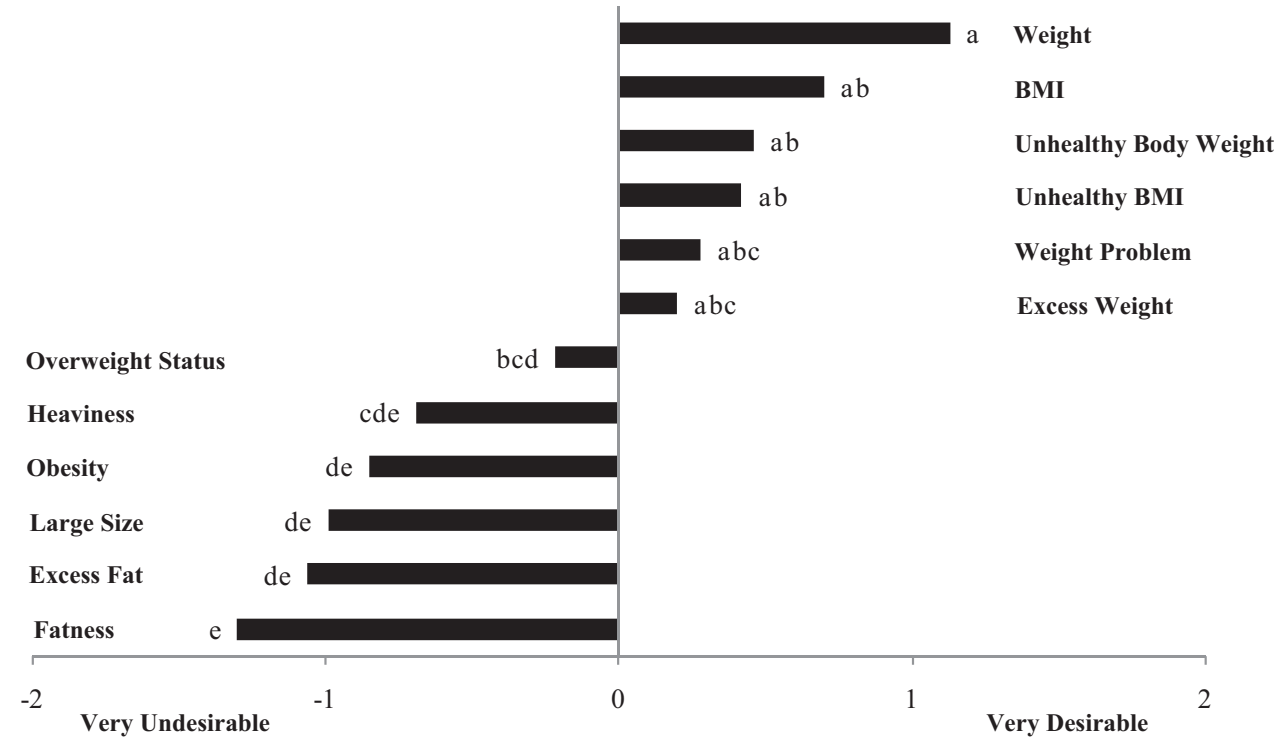

cians (rated as terms they would "maybe" or "probably" use) included "excess weight" (0.60 \pm 1.10$)$, "unhealthy body weight" $(0.52 \pm 1.21)$, "BMI" $(0.24 \pm 1.21)$, and "weight problem" $(0.22 \pm 1.22)$. "Fatness" was least likely to be used by physicians $(-1.74 \pm 0.59)$, although the rating for this term did not significantly differ from "excess fat" $(-1.49 \pm 0.88)$, "heaviness" $(-1.12 \pm 0.99)$, or "large size" (-1.02 \pm 1.05$)$. On average, physicians reported that they would "probably not" or "definitely not" use any of these 4 terms. Comparisons of physician ratings for all 12 terms are summarized in Figure 3.

The physician sample was relatively evenly split in terms of their weight status, with $53 \%$ having a $\mathrm{BMI}$ in the nonoverweight range and $47 \%$ having a $\mathrm{BMI}$ indicative of overweight or obesity. Thus, the likelihood of physicians using each of the 12 terms was compared based on physicians' weight status. There was no difference in normal-weight or overweight/obese physicians' use of any of these weight-related terms with patients.

\section{Discussion}

The current study is the first to assess the preferences of patients' and physicians' use of weightrelated terms while using the same set of terminology for both groups. Patients rated "weight" as the most desirable term for their physician to use to describe overweight or obesity, whereas "fatness" was the least desirable term rated by patients. However, there was notable overlap in patients' ratings of the desirability of the 12 terms provided. Although the pattern/ranking of patients' terminology preferences are consistent with previous research with obese and severely obese patients, ${ }^{13}$ overweight and obese patients in the current sample accepted more terms that could be used by their physicians to describe excess weight. Although "weight" was the only term rated as "desirable" or "very desirable," 5 other terms (including "BMI," "unhealthy body weight," "unhealthy BMI," "weight problem," and "excess weight") were rated favorably by patients (mean ratings between "neutral" and "desirable").

Physicians were most likely to use the term "weight" in discussions with overweight or obese patients, and this term was clearly preferred by physicians when compared with all of the other words offered. In contrast, "fatness," "excess fat," "heaviness," and "large size" were least likely to be used by physicians during clinical encounters. It is encouraging to note that physicians' use of the term "weight" was consistent with patient preferences, and the avoidance of terms such as "excess fat" and "fatness" was also congruent with 
Figure 3. Physicians' reported likelihood of using weight-related terms (scores range from -2 [definitely not use] to +2 [definitely use]). Different letters indicate significant differences between terms using Tukey's multiple comparison procedure to maintain an overall family-wise significance level of .05 . Terms with common letters are not significantly different from each other. BMI, body mass index.

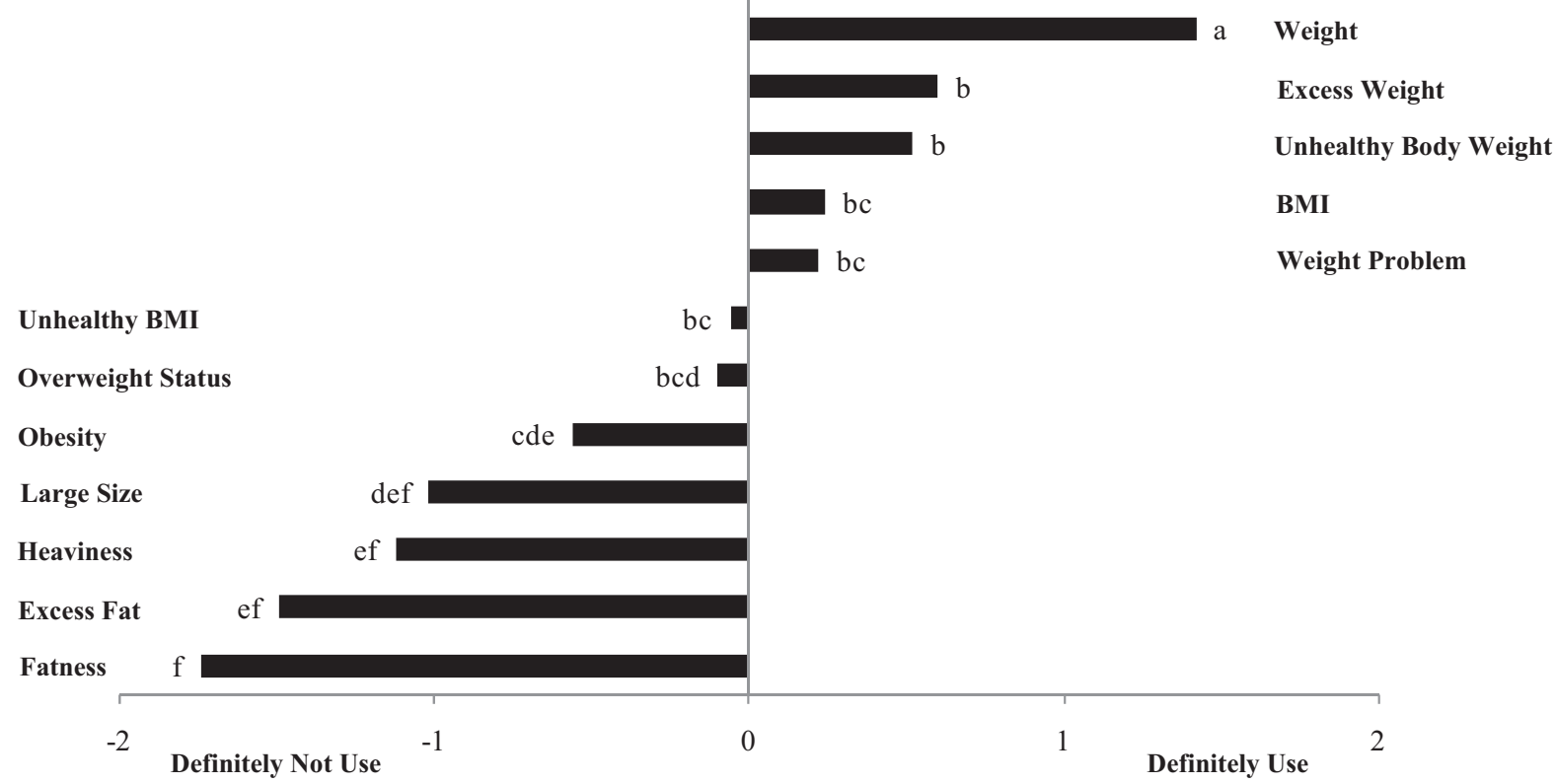

patients' negative reactions to these terms. Similar to comparisons of overweight and obese patients, the weight status of physicians did not influence their reported likelihood of using different weight-related terms with patients.

Few studies have examined the preferences of patients ${ }^{13,16}$ and the parents of pediatric patients ${ }^{14}$ regarding how they would like physicians to broach the topic of obesity. This study expands on this limited research by including a large sample that included both overweight and obese patients who were receiving care in a community-based managed care setting. When comparing patients' preferences based on weight status, overweight and obese patients did not differ in their ratings of any of the terms provided. In fact, the only between-group difference was observed between African-American and white patients' preference for the term "obesity"; white patients found this term more undesirable than African Americans.

Although it is important to explore what terms overweight and obese patients find most appropriate when discussing this topic, it is equally important to understand how physicians actually approach this discussion with their patients. Only one study was identified that examined this issue specifically. ${ }^{16}$ Given several terms and phrases to de- scribe the problem of obesity, physicians most preferred the phrase, "Your weight may be damaging your health." However, this previous study included a very small sample of physicians $(n=19)$, and they were provided with only 8 terms from which to choose.

This topic is particularly worthwhile because physicians can play an important role in the prevention and treatment of obesity, ${ }^{1-3}$ and patients report a desire for greater involvement of their physician in their weight loss efforts. ${ }^{6,7}$ However, this collaborative approach to treatment can only take place after an initiation of the conversation about the patient's need and desire for weight loss. The use of common terminology in patient-provider communications is important to ensure that an effective dialogue occurs. It facilitates the efficient and accurate sharing of information, which is more likely to lead to appropriate diagnoses and treatment plans. ${ }^{15}$ Furthermore, excess weight and related concerns about body image can be an emotionally laden topic, and physicians should acknowledge and deal with patients' emotional responses by effectively communicating respect, empathy, and legitimation. ${ }^{15}$ Physicians' word choices may be an important component of fostering an environment and relationship of mutual respect. Understanding the 
expectations and reactions of both patients and physicians is important to maximize the likelihood of efficient, effective, and satisfying communications between patients and providers about this topic. In fact, preliminary research indicates that the manner in which physicians broach this potentially sensitive topic may influence how patients react emotionally and cognitively to the discussion and advice. ${ }^{16}$

Several limitations to the current study as well as suggestions for future investigation are worth noting. Because the 2 groups responded using a different metric (ie, patients were asked about the desirability of terms whereas physicians were asked how likely they were to use each term), it is not feasible to conduct direct statistical comparisons of physician and patient ratings for each term. Although this study addresses a limitation of previous research by expanding the sample to include overweight individuals, ${ }^{13}$ the proportion of overweight patients was relatively small, so comparisons of overweight and obese patients' preferences should be replicated in future research. Also, the patient sample included only individuals seeking treatment for weight loss, the majority of patients were women, and the sample was relatively well educated. These sample characteristics limit generalizability of the findings, particularly because individuals seeking treatment may have different preferences and comfort levels when discussing their weight status as compared with patients who are not engaged in treatment. Assessing physician behaviors was also novel, although the response rate of physicians was modest. However, the current response rate compared favorably to other studies that included physician surveys. ${ }^{10,19}$ Also, regarding the physician sample, participants were recruited from among clinicians who were affiliated with a medical school. Although the physicians represented communitybased practitioners, this may represent a unique sample, with limited generalizability to other physician populations.

All of the analyses were based on self-reported data rather than observation of recorded clinical encounters. In future study, the use of direct observation or surveys of physicians and patients after an actual office visit may provide more accurate and more detailed information about how this topic is discussed during an encounter. Future research should also adopt a common metric for the assessment of physician and patient preferences and the use of terms to facilitate direct statistical compari- sons between the 2 groups. In addition to the terminology used to describe excess weight, future research should examine other patient and physician attitudes and practices related to weight-loss counseling, such as nonverbal behaviors exhibited during the encounter and the amount of detail and time devoted to these discussions. Finally, one of the most useful extensions and applications of this research will be to examine if the terminology used by physicians and other physician behaviors influence patient outcomes, such as patients' satisfaction with their medical care, their intention to follow their physician's advice, and the success of any weight-loss strategies that have been adopted.

\section{References}

1. Bowerman S, Bellman M, Saltsman P, et al. Implementation of a primary care physician network obesity management program. Obes Res 2001;9(Suppl 4):321S-5S.

2. Galuska DA, Wills JC, Serdula MK, Ford ES. Are health professionals advising obese patients to lose weight? JAMA 1999;282:1576-8.

3. Sciamanna CN, Tate DF, Lang W, Wing RR. Who reports receiving advice to lose weight? Arch Intern Med 2000;160:2334-9.

4. Tsai AG, Wadden TA. Treatment of obesity in primary care practice in the United States: a systematic review. J Gen Intern Med 2009;24:1073-9.

5. Holund U, Thomassen A, Boysen G, et al. Importance of diet and sex in prevention of coronary artery disease, cancer, osteoporosis, and overweight or underweight: a study of attitudes and practices of Danish primary care physicians. Am J Clin Nutr 1997; 65:S2004-6.

6. Potter MB, Vu JD, Croughan-Minihane M. Weight management: what patients want from their primary care physicians. J Fam Pract 2001;50:513-8.

7. Tan D, Zwar NA, Dennis SM, Vagholkar S. Weight management in general practice: what do patients want? Med J Aust 2006;185:73-4.

8. Wadden TA, Anderson DA, Foster GD, et al. Obese women's perceptions of their physicians' weight management attitudes and practices. Arch Fam Med 2000;9:854-60.

9. Bocquier A, Verger P, Basdevant A, et al. Overweight and obesity: knowledge, attitudes, and practices of general practitioners in France. Obes Res 2005;13:787-95.

10. Foster GD, Wadden TA, Makris AP, et al. Primary care physicians' attitudes about obesity and its treatment. Obes Res 2003;11:1168-76.

11. Kushner RF. Barriers to providing nutrition counseling by physicians: a survey of primary care practitioners. Prev Med 1995;24:546-2. 
12. Ruelaz AR, Diefenbach P, MA Simon B, et al. Perceived barriers to weight management in primary care: perspectives of patients and providers. J Gen Intern Med 2007;22:518-22.

13. Wadden TA, Didie E. What's in a name? Patients' preferred terms for describing obesity. Obes Res 2003;11:1140-6.

14. Eneli IU, Kalogiros ID, McDonald KA, Todem D. Parental preferences on addressing weight-related issues in children. Clin Pediatr 2007;46:612-8.

15. Smith RC. Patient-centered interviewing: an evidence-based method. 2nd ed. Philadelphia, PA: Lippincott Williams \& Wilkins; 2002.

16. Tailor A, Ogden J. Avoiding the term "obesity": an experimental study of the impact of doctors' lan- guage on patients' beliefs. Patient Educ Couns 2009; 76:260-4.

17. Teachman BA, Brownell KD. Implicit anti-fat bias among health professionals: is anyone immune? Int J Obes 2001;25:1525-31.

18. Hebl MR, Xu J. Weighing the care: physicians' reactions to the size of a patient. Int J Obes 2001;25:1246-52.

19. Anderson C, Peterson CB, Fletcher L, Mitchell JE, Thuras P, Crow SJ. Weight loss and gender: an examination of physician attitudes. Obes Res 2001;9:257-63.

20. McGuire MT, Wing RR, Klem ML, Lang W, Hill JO. What predicts weight regain in a group of successful weight losers? J Consult Clin Psychol 1999;67:177-85.

21. Stunkard AJ, Albaum JM. The accuracy of selfreported weights. Am J Clin Nutr 1981;34:1593-9. 